\title{
THE THERMAL FORM OF THE PHOTOEFFECT WITH THE DEBYE AND THE WIGNER CRYSTAL
}

\author{
Miroslav Pardy \\ Department of Physical Electronics \\ Masaryk University, \\ Kotlářská 2, 61137 Brno, Czech Republic \\ e-mail:pamir@physics.muni.cz
}

February 17, 2021

\begin{abstract}
We define the photoelectric effect with the specific heat term replacing the work function. The photon propagator involving the radiative correction is also considered. We consider the Debye specific head for the 3D crystal medium, the specific heat for the 2D medium and specific heat for the Wigner crystal.
\end{abstract}

\section{Introduction}

The photoelectric effect is a quantum electromagnetic phenomenon in which electrons are emitted from matter after the absorption of energy from electromagnetic radiation. Frequency of radiation must be above a threshold frequency, which is specific to the type of surface and material. No electrons are emitted with a frequency below of the threshold. The photoelectric effect was theoretically explained by Einstein in his paper in 1905 (Einstein, 1905; 1965) and the term "light quanta" called "photons" was introduced by chemist G. N. Lewis, in 1926. Einstein writes (Einstein, 1905; 1965): In accordance with the assumption to be considered here, the energy of light ray spreading out from point source is not continuously distributed over an increasing space but consists of a finite number of energy quanta which are localized at points in space, which move without dividing, and which can only be produced and absorbed as complete units.

The linear dependence on the frequency was experimentally determined in 1915, when Robert Andrews Millikan showed that Einstein formula

$$
\hbar \omega=\frac{m v^{2}}{2}+W
$$


was correct. Here, $\hbar \omega$ is the energy of the impinging photon, $v$ is the electron velocity measured by the magnetic spectrometer and $W$ is the work function of concrete material. The work function for Aluminium is $4.3 \mathrm{eV}$, for Beryllium $5.0 \mathrm{eV}$, for Lead $4.3 \mathrm{eV}$, for Iron $4.5 \mathrm{eV}$, and so on (Rohlf, 1994). The work function concerns the surface photoelectric effect, where the photon is absorbed by an electron in a band. The theoretical determination of the work function is the problem of the solid state physics. On the other hand, there is the so called atomic photoeffect (Amusia, 1987; Berestetzky et al., 1989), where the ionization energy plays the role of the work function. The system of the ionization energies is involved in the tables of the solid state physics.

The formula (1) is the law of conservation of energy. The classical analogue of the equation (1) is the motion of the Robins ballistic pendulum in the resistive medium.

The idea of the existence of the Compton effect is also involved in the Einstein article. He writes (Einstein, 1905; 1965): The possibility should not be excluded, however, that electrons might receive their energy only in part from the light quantum. However, Einstein was not sure, a priori, that his idea of such process is realistic. Only Compton proved the reality of the Einstein statement.

At energies $\hbar \omega<W$, the photoeffect is not realized. However, the photo-conductivity is the real process. The photoeffect is realized only in medium and with low energy photons, but with energies $\hbar \omega>W$, which gives the Compton effect negligible. For $\hbar \omega \gg W$ the photoeffect is negligible in comparison with the Compton effect. At the same time it is necessary to say that the Feynman diagram of the Compton effect cannot be reduced to the Feynman diagram for photoeffect. In case of the high energy gamma rays, it is possible to consider the process called photoproduction of elementary particles on protons in LHC, or, photo-nuclear reactions in nuclear physics (Levinger, 1960). Such processes are energetically far from the photoelectric effect in solid state physics.

Eq. (1) represents so called one-photon photoelectric effect, which is valid for very weak electromagnetic waves. At present time of the laser physics, where the strong electromagnetic intensity is possible, we know that so called multiphoton photoelectric effect is possible (Delone et al., 1999). Then, instead of equation (1) we can write

$$
\hbar \omega_{1}+\hbar \omega_{2}+\ldots \hbar \omega_{n}=\frac{m v^{2}}{2}+W .
$$

The time lag between the incidence of radiation and the emission of a photoelectron is very small, less than $10^{-9}$ seconds.

The ejected electron has the final plane wave

$$
\psi_{\mathbf{q}}=\frac{1}{\sqrt{V}} e^{i \mathbf{q} \cdot \mathbf{x}}, \quad \mathbf{q}=\frac{\mathbf{p}}{\hbar},
$$

where $\mathbf{p}$ is the momentum of the ejected electron.

The probability of the emission of electron by the electromagnetic wave is of the wellknown form (Davydov, 1976):

$$
d P=\frac{e^{2} p}{8 \pi^{2} \varepsilon_{0} \hbar m \omega}\left|\int e^{i(\mathbf{k}-\mathbf{q}) \cdot \mathbf{x}}(\mathbf{e} \cdot \nabla) \psi_{0} d x d y d z\right|^{2} d \Omega=C|J|^{2} d \Omega,
$$

where the interaction for absorption of the electromagnetic wave is normalized to one photon in the unit volume, $\mathbf{e}$ is the polarization of the impinging photon, $\varepsilon_{0}$ is the dielectric 
constant of vacuum, $\psi_{0}$ is the basic state of an atom. We have denoted the integral in $\|$ by $J$ and the constant before $\|$ by $\mathrm{C}$.

\section{Electrons in magnetic field}

Let us consider the case with electrons in magnetic field as an analog of the Landau diamagnetism. So, we take the basic function $\psi_{0}$ for one electron in the lowest Landau level, as

$$
\psi_{0}=\left(\frac{m \omega_{c}}{2 \pi \hbar}\right)^{1 / 2} \exp \left(-\frac{m \omega_{c}}{4 \hbar}\left(x^{2}+y^{2}\right)\right)
$$

which is solution of the Schrödinger equation in the magnetic field with potentials $\mathbf{A}=(-H y / 2,-H x / 2,0),,($ Drukarev, 1988):

$$
\left[\frac{p_{x}^{2}}{2 m}+\frac{p_{y}^{2}}{2 m}-\frac{m}{2}\left(\frac{\omega_{c}}{2}\right)^{2}\left(x^{2}+y^{2}\right)\right] \psi=E \psi
$$

We have supposed that the motion in the z-direction is zero and it means that the wave function $\exp \left[(i / \hbar) p_{z} z\right]=1$.

So, the main problem is to calculate the integral

$$
J=\int e^{i(\mathbf{K} \cdot \mathbf{x})}(\mathbf{e} \cdot \nabla) \psi_{0} d x d y d z ; \quad \mathbf{K}=\mathbf{k}-\mathbf{q} .
$$

with the basic Landau function $\psi_{0}$ given by the equation (5).

Operator $(\hbar / i) \nabla$ is Hermitean and it means we can rewrite the last integrals as follows:

$$
J=\frac{i}{\hbar} \mathbf{e} \cdot \int\left[\left(\frac{\hbar}{i} \nabla\right) e^{i(\mathbf{K} \cdot \mathbf{x})}\right]^{*} \psi_{0} d x d y d z
$$

which gives

$$
J=i \mathbf{e} \cdot \mathbf{K} \int e^{-i(\mathbf{K} \cdot \mathbf{x})} \psi_{0} d x d y d z
$$

The integral in eq. (9) can be transformed using the cylindrical coordinates with $d x d y d z=\varrho d \varrho d \varphi d z, \quad \varrho^{2}=x^{2}+y^{2}$, which gives for vector $\mathbf{K}$ fixed on the axis $\mathbf{z}$ with $\mathbf{K} \cdot \mathbf{x}=K z$ and with physical condition $\mathbf{e} \cdot \mathbf{k}=0$, expressing the physical situation where polarization is perpendicular to the direction of the wave propagation. So,

$$
J=(i)(\mathbf{e} \cdot \mathbf{q}) \int_{0}^{\infty} \varrho d \varrho \int_{-\infty}^{\infty} d z \int_{0}^{2 \pi} d \varphi e^{-i K z} \psi_{0}
$$

Using

$$
\psi_{0}=A \exp \left(-B \varrho^{2}\right) ; \quad A=\left(\frac{m \omega_{c}}{2 \pi \hbar}\right)^{1 / 2} ; \quad B=\frac{m \omega_{c}}{4 \hbar},
$$

the integral (10) is then

$$
J=(-\pi i) \frac{A}{B}(\mathbf{e} \cdot \mathbf{q}) \int_{-\infty}^{\infty} e^{-i K z} d z=(-\pi i) \frac{A}{B}(\mathbf{e} \cdot \mathbf{q})(2 \pi) \delta(K) .
$$

Then, 


$$
d P=C|J|^{2} d \Omega=4 \pi^{4} \frac{A^{2}}{B^{2}} C(\mathbf{e} \cdot \mathbf{q})^{2} \delta^{2}(K) d \Omega .
$$

Now, let be the angle $\Theta$ between direction $\mathbf{k}$ and direction $\mathbf{q}$, and let be the angle $\Phi$ between planes $(\mathbf{k}, \mathbf{q})$ and $(\mathbf{e}, \mathbf{k})$. Then,

$$
(\mathbf{e} \cdot \mathbf{q})^{2}=q^{2} \sin ^{2} \Theta \cos ^{2} \Phi
$$

So, the differential probability of the emission of photons from the graphene (Pardy, 2010) in the strong magnetic field is as follows:

$$
d P=\frac{4 e^{2} p}{\pi \varepsilon_{0} m^{2} \omega \omega_{c}}\left[q^{2} \cos ^{2} \Theta \sin ^{2} \Phi\right] \delta^{2}(K) d \Omega ; \quad \omega_{c}=\frac{|e| H}{m c} .
$$

We can see that our result differs form the result for the original photoelectric effect which involves still the term

$$
\frac{1}{\left(1-\frac{v}{c} \cos \Theta\right)^{4}}
$$

which means that the most intensity of the classical photoeffect is in the direction of the electric vector of the electromagnetic wave $(\Phi=\pi / 2, \Theta=0)$. While the nonrelativistic solution of the photoeffect in case of the Coulomb potential was performed by Stobbe (1930) and the relativistic calculation by Sauter (Sauter, 1931), the general magnetic photoeffect (with electrons moving in the magnetic field and forming atom) was not still performed in a such simple form. The delta term $\delta \cdot \delta$ represents the conservation law $|\mathbf{k}-\mathbf{q}|=0$ in our approximation.

So, we have calculated only the process which can be approximated by the Schrödinger equation for an electron orbiting in magnetic field.

\section{Photon with the radiative correction}

The photoeffect with the dressed photon is the process, where the dressed photon is taken with the radiative correction in the form of the virtual electron-positron pair.

We have shown that such approach to the photon leads to the modification he photon propagator. According to Dittrich (1978) and Schwinger (1973), the photon propagator with radiative correction is in the momentum representation of the form:

$$
\tilde{D}(k)=D(k)+\delta D(k)
$$

or,

$$
\begin{gathered}
\tilde{D}(k)=\frac{1}{|\mathbf{k}|^{2}-n^{2}\left(k^{0}\right)^{2}-i \epsilon}+ \\
+\int_{4 m^{2}}^{\infty} d M^{2} \frac{a\left(M^{2}\right)}{|\mathbf{k}|^{2}-n^{2}\left(k^{0}\right)^{2}+\frac{M^{2} c^{2}}{\hbar^{2}}-i \epsilon}
\end{gathered}
$$

where the last term in equation (18) is derived on the virtual photon condition 


$$
|\mathbf{k}|^{2}-n^{2}\left(k^{0}\right)^{2}=-\frac{M^{2} c^{2}}{\hbar^{2}}
$$

where $n$ is the index of refraction of the medium. The weight function $a\left(M^{2}\right)$ has been derived in the following form (Dittrich, 1978; Schwinger, 1973):

$$
a\left(M^{2}\right)=\frac{\alpha}{3 \pi} \frac{1}{M^{2}}\left(1+\frac{2 m^{2}}{M^{2}}\right)\left(1-\frac{4 m^{2}}{M^{2}}\right)^{1 / 2} .
$$

The x-representation of $D(k)$ in eq. (18) is as follows:

$$
D_{+}\left(x-x^{\prime}\right)=\int \frac{(d k)}{(2 \pi)^{4}} e^{i k\left(x-x^{\prime}\right)} D(k) .
$$

Or,

$$
\begin{gathered}
D_{+}\left(x-x^{\prime}\right)=\int \frac{(d k)}{(2 \pi)^{4}} \frac{e^{i k\left(x-x^{\prime}\right)}}{\left|\mathbf{k}^{2}\right|-n^{2}\left(k^{0}\right)^{2}-i \epsilon}= \\
=\frac{i}{c} \frac{1}{4 \pi^{2}} \int_{0}^{\infty} d \omega \frac{\sin \frac{n \omega}{c}\left|\mathbf{x}-\mathbf{x}^{\prime}\right|}{\left|\mathbf{x}-\mathbf{x}^{\prime}\right|} e^{-i \omega\left|t-t^{\prime}\right|} .
\end{gathered}
$$

Now, with regard to the definition of x-representation (21) and (22) of the $D_{+}\left(x-x^{\prime}\right)$, we get the x-representation of the $\delta D_{+}$in the following form:

$$
\begin{gathered}
\delta D_{+}\left(x-x^{\prime}\right)=\frac{i}{c} \frac{1}{4 \pi^{2}} \int_{4 m^{2}}^{\infty} d M^{2} a\left(M^{2}\right) \times \\
\times \int d \omega \frac{\sin \left[\frac{n^{2} \omega^{2}}{c^{2}}-\frac{M^{2} c^{2}}{\hbar^{2}}\right]^{1 / 2}\left|\mathbf{x}-\mathbf{x}^{\prime}\right|}{\left|\mathbf{x}-\mathbf{x}^{\prime}\right|} e^{-i \omega\left|t-t^{\prime}\right|} .
\end{gathered}
$$

The function (23) differs from the the original function $D_{+}$especially by the factor

$$
\gamma=\left(\frac{\omega^{2} n^{2}}{c^{2}}-\frac{M^{2} c^{2}}{\hbar^{2}}\right)^{1 / 2}
$$

and by the additional mass-integral which involves the radiative corrections to the original photon processes. It was easily shown in case of the Cerenkov effect by author (Pardy, 1994).

So, to involve the photoelectric effect with the dressed photon with electron positron pair we replace the wave function of photon $\exp (i \mathbf{k} \cdot \mathbf{x})$ by the function involving the radiative correction factor as follows:

$$
e^{i \mathbf{k} \cdot \mathbf{x}} \rightarrow \int_{4 m^{2}}^{\infty} d M^{2} a\left(M^{2}\right) e^{i \boldsymbol{\kappa} \cdot x}
$$

where $\boldsymbol{\kappa} \cdot \mathbf{x}=\gamma|k||x| \cos \varphi$.

The probability of the emission of electron by the electromagnetic wave is given by eq. (4).

So, the main problem is to calculate the integral

$$
J=\int e^{i(\boldsymbol{\kappa} \cdot \mathbf{x})}(\mathbf{e} \cdot \nabla) \psi_{0} d x d y d z ; \quad \mathbf{K}=\boldsymbol{\kappa}-\mathbf{q} .
$$


with the basic Landau function $\psi_{0}$ given by the equation (5).

Then, the differential probability of the emission of photons from the plane in the strong magnetic field is as follows:

$$
d P=\frac{4 e^{2} p}{\pi \varepsilon_{0} m^{2} \omega \omega_{c}} \int_{4 m^{2}}^{\infty} d M^{2} a\left(M^{2}\right)\left[q^{2} \cos ^{2} \Theta \sin ^{2} \Phi\right] \delta^{2}(K) d \Omega ; \quad \omega_{c}=\frac{|e| H}{m c} .
$$

We can see that our result differs form the result (15) by the mass term and by the $\operatorname{argument}$ in the $\delta$-function. The delta term $\delta \cdot \delta$ represents the conservation law $|\boldsymbol{\kappa}-\mathbf{q}|=0$ in our approximation. The dressed photon was here considered as the photon composed from the electron-positron pair. It is not excluded that the photoelectric experiments with the dressed photon is related to the experiments with the Vavilov-Cherenkov phenomenon in metal nanofilms (Pardy, 2007, 2010, 2011; Zuev, 2009).

\section{The specific heat form of the photo-electric effect with the Debye crytal}

It is well known that the Einstein approach the photoelectric effect is today considered as the product of the naive thinking. More realistic approach is one, where the work function is replaced by the thermal characteristic of medium under irradiation by photons. So we replace the old Einstein equation (1) by the following one:

$$
\hbar \omega=\frac{m v^{2}}{2}+C_{V} \Delta T
$$

where $C_{V}$ is the specific heat of medium and $\Delta T$ is the the change of temperature which gives the information of the interaction of the energy of moving electron with the medium. In other words, the energy loss of electron in medium is detected by the temperature state of the crystal.

We know, that in case of the tree-dimensional crystal medium the term $C_{V}$ is givem by the Debye formula (Debye, 1912a; 1912b):

$$
C_{V}=3 N k \int d \omega \varrho(\omega)\left(\frac{\hbar \omega}{2 k T}\right)^{2}\left(\sinh \frac{\hbar \omega}{2 k T}\right)^{-2}
$$

where $\varrho(\omega)$ is the density of states with the frequency $\omega$.

In case of the two-dimensional crystal, it is possible to show that the term $C_{V}$ is as follows

$$
C_{V}=\left(\frac{\partial U}{\partial T}\right)_{V}
$$

where $U$ is obtained by calculation in the form (Rumer et al., 1977):

$$
U=\frac{4}{3} N \theta\left(1+3\left(\frac{T}{\theta}\right)^{3} \int_{0}^{\theta / T} \frac{x^{2} d x}{e^{x}-1}\right), \theta=\hbar \omega\left(\frac{N}{\pi \sigma}\right)^{1 / 2},
$$

where $\sigma$ is the area of the crystal. 


\section{The specific heat form of the photo-electric effect with the Wigner crystal}

A Wigner crystal is the solid (crystalline) phase of electrons (Wigner, 1934). A gas of electrons moving in neutralizing background crystallize and form a lattice if the electron density is less than a critical value. In this case the potential energy dominates the kinetic energy at low densities, so the detailed spatial arrangement of the electrons becomes important. To minimize the potential energy, the electrons form a bodycentered cubic lattice in 3D, a triangular lattice in 2D and an evenly spaced lattice in 1D. Most experimentally observed Wigner clusters exist due to the presence of the external potential trap. A crystalline state of the $2 \mathrm{D}$ electron gas can also be realized by applying a sufficiently strong magnetic field. However, it is still not clear whether it is the Wigner-crystallization that has led to observation of insulating behavior in magnetotransport measurements on 2D electron systems, since other candidates are present, such as Anderson localization. More generally, a Wigner crystal phase can be occurring in non-electronic systems at low density. In contrast, most crystals melt as the density is lowered (Wigner, 1934).

The specific heat of such medium was derived by Usov et al. (1980) in the case of a strong magnetic field $\omega_{c} \gg \omega_{p}$, where $\omega_{c}$ is the cyclotron frequency and $\omega_{p}$ is the plasma frequency of the Wigner crystal . The Debye temperature of a Wigner lattice in the absence of a magnetic field is $\theta \sim \hbar \omega_{p}$ (Usov et al., 1980).

In the Debye approximation, the specific heat of the system formed by the Wigner lattice was obtained as follows (Usov et al., 1980):

$$
C_{V} \sim \frac{N}{V}\left(\frac{T \hbar \omega_{c}}{\theta^{2}}\right) \int_{0}^{\theta^{2} / T \hbar \omega_{c}} \frac{x^{3 / 2} e^{x} d x}{\left(e^{x}-1\right)^{2}}
$$

Thus at $T \leq \theta^{2} / \hbar \omega_{c}$, where $\omega_{c}$ is the cyclotron frequency, the specific heat of the system $C_{V}$ is $\sim T^{3 / 2} B^{3 / 2}, B$ being the magnetic induction (Usov et al., 1980). Carr has shown that the Debye temperature of a WL in the absence of a magnetic field is $\theta \sim \hbar \omega_{p}$ (Carr, Jr., 1961).

\section{Discussion}

The photoelectric effect is the integral part of the more general effect of the interaction of photons with the noncrystalline and crystalline medium. The starting point of the problem by author is the author diploma work (Pardy, 1965).

Of course, from the historical point of view, the starting point of interaction of light with matter is the Newton Optics (Newton, 1704), where he introduced the light as the system of photons interacting with medium composed from the Gassendi atoms.

Newton proposed that light was composed of corpuscles that traveled in straight lines. That worked fine for reflection, because the bouncing of either particles or waves off a planar surface follows the same law of reflection. But to explain refraction, he had to presume that the particles traveled faster in a more optically dense material. But Foucault's 1850 experiment showed that light traveled more slowly in such media, so that version of a particle theory of light had to be set aside.

Nevertheless, the Newton photo-atomic equation is as follows: 


$$
\text { Energy }(\text { photon })=\operatorname{Energy}(\text { atom })+\operatorname{Energy}(\text { loss of atom in medium })
$$

Let us remark that the last equation (32) was never published by Isaac Newton.

The Einstein formula was published after some centuries in the naive form:

$$
\hbar \omega=\frac{m v^{2}}{2}+W
$$

where $W$ is the work function.

The experimental form of the photoelectric equation with the large application in the solid state physics, superconductivity, astrophysics, condensed matter physics, gas physics and so on, is given here by our equation

$$
\hbar \omega=\frac{m v^{2}}{2}+C_{V} \Delta T
$$

where $C_{V}$ is the specific heat at the constant volume of solid crystals, condensed matter and liquids. The most interesting case is the application of the equation for the Wigner crystal. It is not excluded that our equation (34) will play the substantial role in the modern physics, cosmology, quantum chemistry and the quantum biophysics of photosynthesis.

\section{REFERENCES}

Amusia, M. Ya. Atomic photoeffect, (Nauka, Moscow, 1987). (in Russian).

Berestetzky, V. B., Lifshitz, E. M. and Pitaevskii, L. P. Quantum electrodynamics, (Moscow, NAUKA, 1989). (in Russian).

Carr, Jr., W. J. (1961). Energy, Specific Heat, and Magnetic Properties of the LowDensity Electron Gas, Phys. Rev. 122, 1437.

Davydov, A. S. Quantum mechanics; 2-nd ed., (Pergamon Press, Oxford, New York, 1976).

Debye, P. (1912a). Zur Theorie der spezifischen Wärmen. Annalen der Physik, 39 (4), 789-839.

Debye, P. (1912b). Zur Theorie der spezifischen Wärmen. Annalen der Physik, 344 (14), 789-839

Delone, N. B. and Krainov, V. P. Multiphoton processes in atoms, 2nd enl. and updated ed., (Springer series on atoms + plasmas, 1999).

Dittrich, W. (1978). Source methods in quantum field theory, Fortschr. Phys. 26, 289.

Drukarev, G. F. Quantum mechanics, (St. Petersburgh University, 1988). (in Russian).

Einstein, A., (1905). Über einen die Erzeugung und Verwandlung des Lichtes betreffenden heuristichen Gesichtspunkt, Annalen der Physik, 17, 132.

Einstein, A., (1965). On the Heuristic Viewpoint Concerning the Production and Transformation of Light, AJP, 33, No. 5, May (1965). (The English translation of Einstein article from 1905). 
Levinger, J. S. Nuclear photo-desintegration. (Oxford University Press, 1960). (in Russian).

Newton, I. Opticks: or, a treatise of the reflexions, refractions, inflexions and colours of light. Also two treatises of the species and magnitude of curvilinear figures. (London, 1704).

Pardy, M. (1965). The interaction of Light with crystal, Diploma work of J. E. Purkynje University, Brno. (Unpublished).

Pardy, M. (1994). The Cherenkov effect with radiative corrections, Physics Letters B 325, 517 .

Pardy, M. (2007). The photoeffect at the low temperature graphene in the strong magnetic field, hep-ph/0707.2668v2.

Pardy, M. The photoelectric effect on graphene; Scientific Research and Essays, Vol. 5(12), pp. 1571-1575, 18 June, 2010 Available online at http: www.academicjournals.org/SRE ISSN 1992-2248, 2010 Academic Journals

Pardy, M. Photoeffect in graphene and axion detection by graphene; In: Graphene Simulation; Edited by Jian Ru Gong, (Published by InTech , Janeza Trdine 9, 51000 Rijeka, Croatia, ISBN 78-953-307-556-3, 2011).

Rohlf, J. W. Modern Physics from a to $Z^{0}$, (John Willey \& Sons, Inc., New York, 1994).

Rumer, Yu. B. and Ryvkin, M. Sch.. Thermodynamics, Statistical Physics and Kinetics, (NAUKA, Moscow, 1980). (in Russian).

Sauter, F., (1931). Über Atomaren Photoeffekt bei Grosser Härte der Anwegenden Strahlung, Ann. der Phys. 9, 217.

Schwinger, J. (1973). Particles, Sources and Fields, Vol. 2 (Addison-Wesley Publ. Comp., Reading, Mass).

Stobbe, M., (1930). Zur Quantenmechanik photoelektrischer Prozesse, Ann. der Phys. 7,661 .

Wigner, E.P., (1934). On the interaction of electrons in metals, Physical Review, 46, pp. 1002-1011.

Usov, N. A. , Grebenshchikov, Yu. B. and Ulinich, F. R. (1980). Three-dimensional Wigner crystal in a magnetic field, Sov. Phys. JETP 51(1), Jan.

Zuev, V. S. (2009). Vavilov-Cherenkov phenomenon in metal nanofilms, arXiv: 0907.1145, [Optics (physics-optics)]. 